\title{
$\mathrm{H}$ \\ овые возможности терапии атопического дерматита у детей и взрослых
}

\author{
Д.В. Прошутинская, М.М. Бутарева, Л.А. Иноятова
}

ФГБУ «Государственный научный центр дерматовенерологии и косметологии» Минздрава России 107076, Москва, ул. Короленко, д. 3, стр. 6

\footnotetext{
Цель. Изучение эсрфективности и безопасности наружного применения такролимуса в фрорме 0,03\% и 0,1\% мази у больных атопическим дерматитом средней и тяжелой степени.

Материал и методы. Под наблюдением находились 32 пациента в возрасте от 2 до 64 лет, больных атопическим дерматитом. Первая группа пациентов состояла из 17 детей (7 девочек , 10 мальчиков) в возрасте от 2 до 15 лет. Вторую группу составили 15 пациентов (8 женщин, 7 мужчин) в возрасте от 18 до 64 лет. Все больные страдали атопическим дерматитом средней и тяжелой степени тяжести. Больные обеих групп получали наружную терапию такролимусом в фрорме 0,03\% мази для детей и 0,1\% мази для взрослых. Расчет потребляемого в сутки количества мази производился из расчета количества единиц FTU (Finger Tip Unit) для отдельных областей тела. Для объективной оценки степени тяжести клинических проявлений атопического дерматита и эффективности проводимой терапии у всех наблюдаемых больных был использован индекс SCORAD (Severity Scoring of Atopic Dermatitis).

Результаты. Терапия привела к статистически значимому уменьшению медианы индекса SCORAD c 67,2 (54,8; 70,4) до 14,0 (3,5; 22,1$)$ баллов $(p<0.0001)$ в 1-й группе и с $52(43,5 ; 75,5)$ до 11,7 $(0,0 ; 26,2)$ балла $(p<0.0001)$ во 2-й группе. Клиническое выздоровление достигнуто у $30 \%$ детей и у $40 \%$ взрослых.

Заключение. Применение мази Протопик в лечении больных атопическим дерматитом со среднетяжелой и тяжелой степенью показало высокую эфрфективность, что проявилось в положительном терапевтическом влиянии на течение заболевания. При этом отмечалось отсутствие побочных эффректов, характерных для топических кортикостероидных препаратов, что значительно расширяет возможности лечения пациентов с данным дерматозом.
}

Ключевые слова: атопический дерматит, такролимус, дети, взрослые, эффективность лечения.

Контактная информация: detstvo@cnikvi.ru. Вестник дерматологии и венерологии 2013; (3): 78—82.

\section{New potential of the treatment of atopic dermatitis in children and adults}

D.V. Proshutinskaya, M.M. Butareva, L.A. Inoyatova

State Research Center of Dermatovenereology and Cosmetology, Ministry of Healthcare of the Russian Federation Korolenko str. 3, bldg 6, Moscow, 107076, Russia

Goal. To study the efficacy and safety of local administration of Tacrolimus in the form of $0.03 \%$ and $0.1 \%$ ointment for patients with moderate to severe atopic dermatitis.

Materials and methods. The study involved 32 patients suffering from atopic dermatitis and aged 2-64. The first group comprised 17 children aged $2-15$ (7 girls and 10 boys). The second group comprised 15 patients ( 8 female and 7 male) aged 18 -64. All of the patients suffered from moderate to severe atopic dermatitis. Patients from both treatment groups received external therapy in the form of $0.03 \%$ of ointment for children and $0.1 \%$ of ointment for adults. The daily ointment amount was calculated as FTU (Finger Tip Units) for separate body parts. To ensure objective assessment of the severity of clinical manifestations of atopic dermatitis and treatment efficacy, SCORAD (Severity Scoring of Atopic Dermatitis) score was used for all of the patients.

Results. The treatment resulted in a statistically significant reduction in the medial SCORAD index from $67.2(54.8 ; 70.4)$ points to 14.0 $(3.5 ; 22.1)$ points $(p<0.0001)$, Group 1 , and from $52(43.5 ; 75.5)$ points to $11.7(0.0 ; 26.2)$ points $(p<0.0001)$, Group 2. Clinical recovery was achieved in $30 \%$ of children and $40 \%$ of adults.

Conclusion. Protopic ointment for the treatment of patients with moderate to severe atopic dermatitis proved to be highly efficient, which was confirmed by a positive therapeutic effect on the course of the disease. There were no adverse effects characteristic of topical corticosteroids, which widens horizons in terms of treating patients with this type of dermatosis.

Key words: atopic dermatitis, Tacrolimus, children, adults, treatment efficacy. 
Атопический дерматит (АтД) остается одним из самых распространенных хронических дерматозов как среди взрослых, так и среди детей, поражая до 10$20 \%$ популяции [1, 2]. Несмотря на то что в последние десятилетия ученые далеко продвинулись в понимании генетических аспектов и механизмов патогенеза АтД, методы его лечения все еще недостаточно совершенны. Терапия в первую очередь направлена на уменьшение клинических проявлений заболевания, предотвращение рецидивов и снижение степени их тяжести. Обеспечение такого контроля над заболеванием, особенно у больных с тяжелыми формами, возможно только при проведении долговременной терапии. Традиционно основными в лечении АтД являются топические глюкокортикостероидные средства [3, 4]. Многолетний опыт применения препаратов этой группы подтверждает их высокую эффрективность [5-7]. В то же время риск развития таких нежелательных явлений, как атрофия кожи, телеангиэктазии, стероидные угри, гипертрихоз, повышение внутриглазного давления при нанесении на область век, ограничивает их продолжительное применение, особенно у детей [8].

Появление новой группы наружных средств - топических ингибиторов кальциневрина, в частности препарата Протопик - значительно расширило возможности терапии АтД у пациентов со среднетяжелым и тяжелым течением [9-11]. Препараты данной группы оказывают иммунорегуляторное действие на патологические реакции в коже больных АтД посредством избирательного блокирования внутриклеточного протеина кальциневрина. Последний ответственен за транслокацию в ядро нуклеарного фактора активированных Т-лимфоцитов и активацию генов, кодирующих различные провоспалительные цитокины [12]. Принципиальные различия в механизме действия топических ингибиторов кальциневрина и ТГКС позволяют использовать препараты этой группы в течение продолжительного времени у детей и взрослых, больных АтД, в том числе и на участках с тонкой кожей, не опасаясь развития стероидоподобных нежелательных явлений.

Целью настоящего исследования явилось изучение эфрфективности и безопасности местного применения такролимуса в форме 0,03\% и 0,1\% мази у больных АтД средней и тяжелой степени.

\section{Материал и методы}

Под клиническим наблюдением в отделениях детской и клинической дерматологии ФГБУ «ГНЦДК» Минздрава России находились 32 больных АтД. Возраст больных составил от 2 до 64 лет.

Первая группа пациентов включала 17 детей (7 девочек, 10 мальчиков) в возрасте от 2 до 15 лет. Один ребенок, у которого не отмечалось положительной динамики в течение недели после начала лечения, по настоянию родителей был выведен из исследования и при статистической обработке результатов не учитывался. Вторую группу составили 15 взрослых (8 женщин, 7 мужчин) в возрасте от 18 до 64 лет. У всех больных была средняя и тяжелая степень тяжести АтД. Продолжительность заболевания составила от 2 до 33 лет (в среднем 14,7 года). Пациенты или их представители (в случае детей) констатировали частоту рецидивов заболевания не менее 4-6 раз в год, регулярное применение наружных кортикостероидных средств.

Среди пациентов детского возраста у преобладающего большинства - 15 (88\%) - отмечалась эритематосквамозная форма с лихенификацией. У одного больного - лихеноидная форма, у одного - пруригинозная. У всех взрослых пациентов отмечалась лихеноидная форма АтД.

Больные обеих групп получали наружную терапию такролимусом в форме 0,03\% мази Протопик для детей и $0,1 \%$ мази Протопик для взрослых. Не ранее чем через 2 ч. после нанесения исследуемого препарата больным рекомендовалось использовать наружные увлажняющие средства.

Системная терапия в обеих группах включала антигистаминные препараты (Супрастин, Фенкарол, Зиртек), дезинтоксикационные (Энтеросгель, Лактофильтрум, Тиосульфат натрия) и гипосенсибилизирующие средства (хлорид кальция, глицерофоссрат кальция).

Расчет потребляемого в сутки количества мази производился из расчета количества единиц FTU (Finger Tip Unit) для отдельных областей тела. FTU — количество мази из тубы с диаметром отверстия 5 мм, покрывающее дистальную фалангу указательного пальца взрослого человека. Принималось во внимание, что 1 FTU равна приблизительно 0,5 г. Зная процент пораженной кожи и используя правило девяток, составлялась пропорция, в результате чего получали необходимое значение в граммах.

Для объективной оценки степени тяжести клинических проявлений АтД и эффрективности проводимой терапии использовали индекс SCORAD (Severity Scoring of Atopic Dermatitis). Критерием эффрективности лечения служило достижение положительных клинических результатов в виде уменьшения или полного разрешения зуда и признаков воспаления на коже.

Значения индекса SCORAD от 20 до 60 баллов coответствовали средней степени тяжести заболевания, более 60 - тяжелой.

Клиническое выздоровление - полное разрешение кожного процесса

Значительное улучшение - снижение величины индекса SCORAD не менее чем на 75\% по сравнению с исходными данными.

Улучшение - снижение величины индекса SCORAD менее чем на $75 \%$, но более $25 \%$ по сравнению с исходными данными. 
Без изменений - снижение величины индекса SCORAD менее чем на 25\% или отсутствие изменений по сравнению с исходными данными.

Ухудшение - усиление вовлечения кожных покровов в патологический процесс по сравнению с исходными данными.

Статистический анализ проводился в системе статистического анализа SAS (программный пакет SAS Institute, США, версия 9.1.3 для Windows XP). Описание количественных признаков, соответствующих нормальному распределению, представлено в виде среднего значения \pm стандартное квадратическое отклонение, признаки, отличающиеся от нормального распределения, - в виде медианы, 25 и 75\% квартилей. Для сравнения количественных признаков использовались критерии Манна - Уитни и Вилкоксона.

\section{Результаты и обсуждение}

Перед началом лечения в 1-й группе детей средняя и тяжелая степень АтД отмечалась у 7 (41\%) и 10 (59\%) больных соответственно. Значения индекса SCORAD составляли от 40,2 до 81,3 балла (в среднем 63,4 \pm 12 баллов). Во 2-й группе старше 18 лет средняя степень тяжести отмечалась у 9 (60\%) больных, тяжелая - у 6 (40\%). Величина индекса SCORAD варьировалась от 26,3 до 87,2 балла, (в среднем - 57,1 \pm 18,4 балла). Статистически значимых различий по величине индекса SCORAD до лечения у детей и взрослых больных АтД не отмечалось $(p \geq 0,05)$.

Пациентам детского возраста препарат с содержанием действующего вещества такролимуса 0,03\% назначался в режиме 2 раза в сутки в течение 3 нед., затем при положительной динамике кожного процес- са и необходимости продолжения терапии кратность нанесения составляла один раз в сутки до полного разрешения высыпаний. Взрослым больным был рекомендован режим применения мази с концентрацией действующего вещества 0,1\% два раза в сутки до достижения улучшения, затем с концентрацией 0,03\% до достижения клинического выздоровления.

Курс лечения в 1-й группе составил от 10 до 40 дней (в среднем 18 дней). Наступление улучшения в виде уменьшения воспаления на коже и снижения интенсивности зуда констатировалось больными на 2-7-й день, в среднем на 5-й день лечения. К концу первой недели лечения медиана индекса SCORAD равнялась 30,1 балла (более чем в 2 раза ниже исходной величины - 67,2 балла; рис. 1). Клинически отмечалось значительное уменьшение выраженности эритемы, папулезных высыпаний, инфильтрации в очагах воспаления (рис. 2), а также уменьшение зуда и улучшение сна у больных. В процессе лечения ухудшения не отмечалось ни у одного пациента. Клиническое выздоровление наступило у 5 (30\%) больных, значительное улучшение наблюдалось у 6 (35\%), улучшение - у 6 (35\%) пациентов. Отмечен выраженный положительный ответ на лечение при локализации очагов высыпаний на лице, в том числе в периоральной области (рис. 3). После окончания курса терапии отмечалось статистически значимое уменьшение медианы индекса SCORAD с $67,2(54,8 ; 70,4)$ до $14,0(3,5 ; 22,1)$ баллов $(p<0,0001)$. При этом значение общего курсового потребления мази варьировало от 1,5 до 70 граммов (медиана 30 г). Среднесуточный показатель расхода мази составил $1,9 \pm 1,4$ г. Лечение все больные переносили хорошо. Из нежелательных явлений у 4 (23\%) пациентов отме-

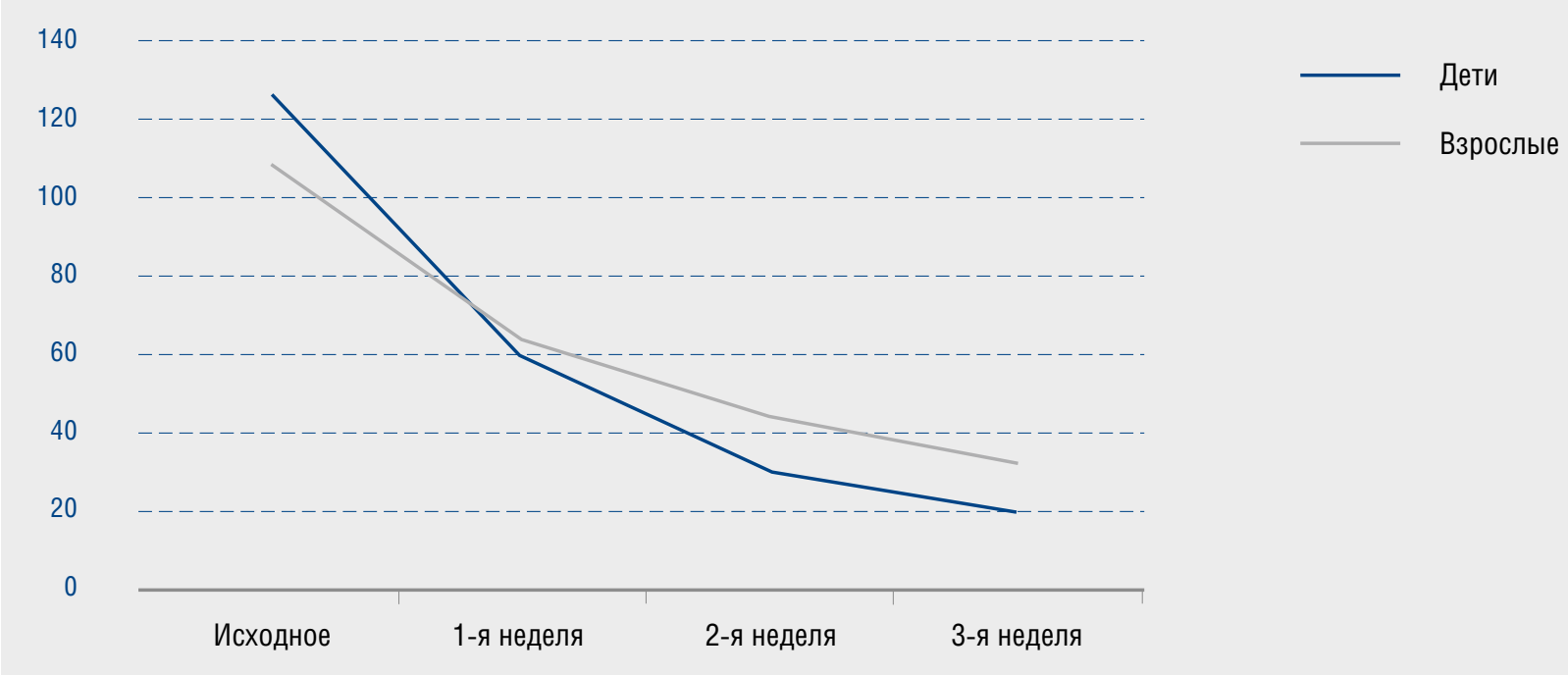

Pис. 1. Динамика величины индекса SCORAD в процессе лечения в группах детей и взрослых 


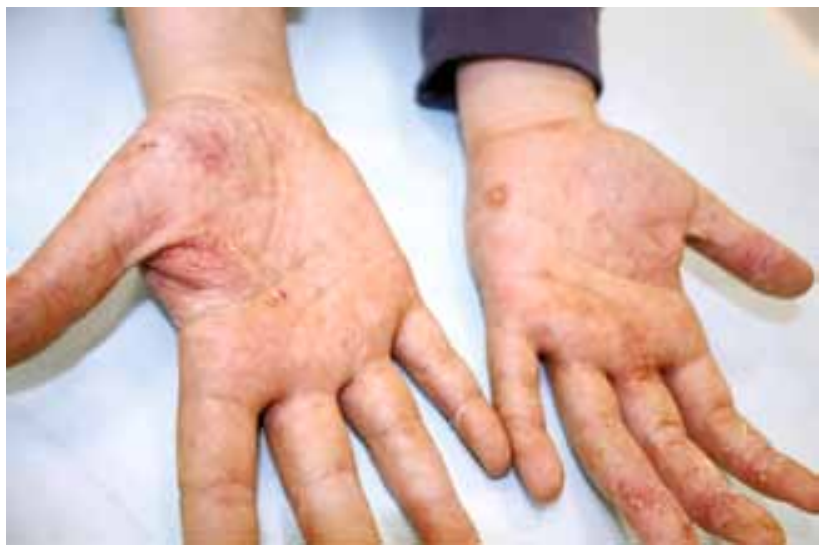

a

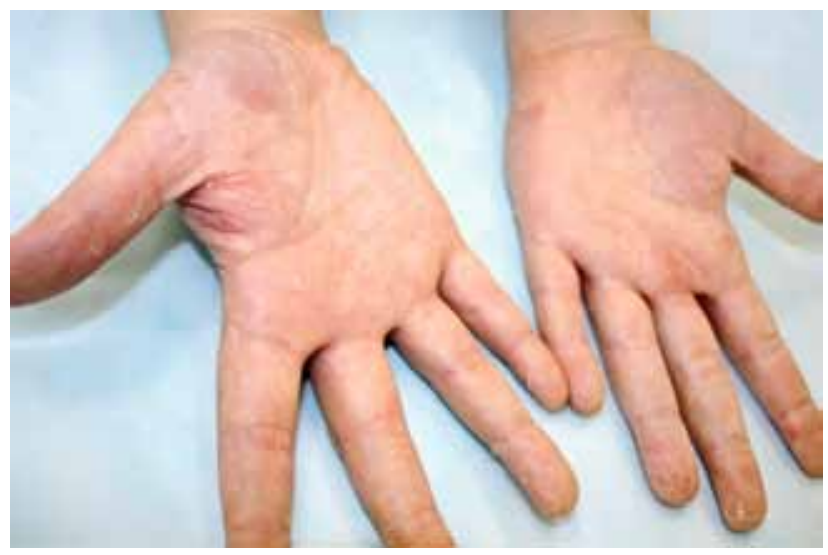

$\sigma$

Рис. 2. Мальчик 8 лет, больной АтД: а - до лечения; б - на 7-й день терапии 0,03\% такролимусом

чалось легкое жжение и незначительный зуд при нанесении средства, самопроизвольно разрешающиеся в течение нескольких дней.

Продолжительность терапии больных 2-й группы варьировала от 15 до 40 дней, составляя в среднем 25 дней. Наступление улучшения больными констатировано на 5-15-й день, в среднем на 9-й день лечения. Ухудшения состояния на фоне терапии такролимусом не отмечалось ни у одного пациента. Клини- ческое выздоровление было достигнуто у 6 больных (40\%), значительное улучшение - у 3 (20\%), улучшение - у $6(40 \%)$. Если до начала лечения медиана величины индекса SCORAD составляла 52 балла, то к концу первой недели после начала терапии ее значение снизилось до 39,2 балла, а к концу 14-го дня лечения равнялось 20 баллам, т. е. уменьшилось более чем в 2 раза (см. рис.1). После окончания лечения отмечалось статистически значимое уменьшение медианы

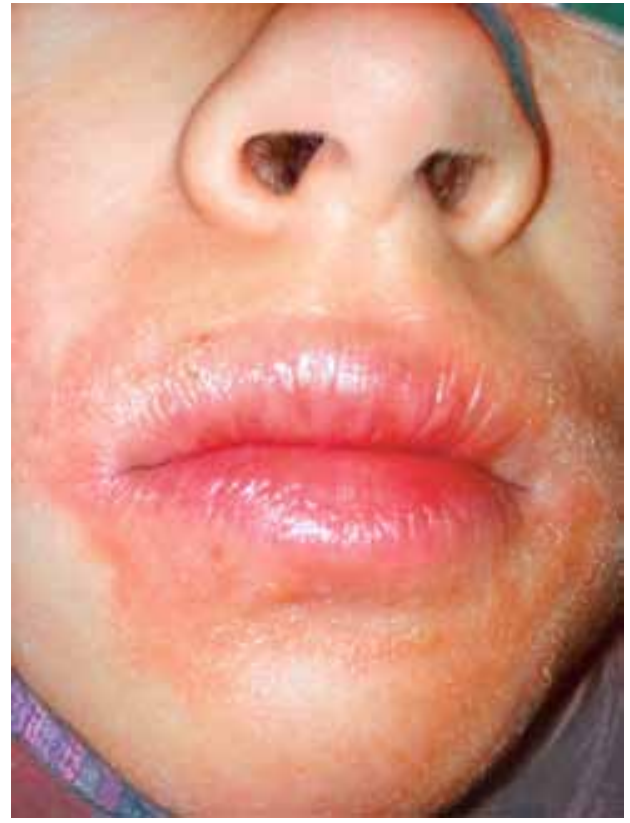

$a$

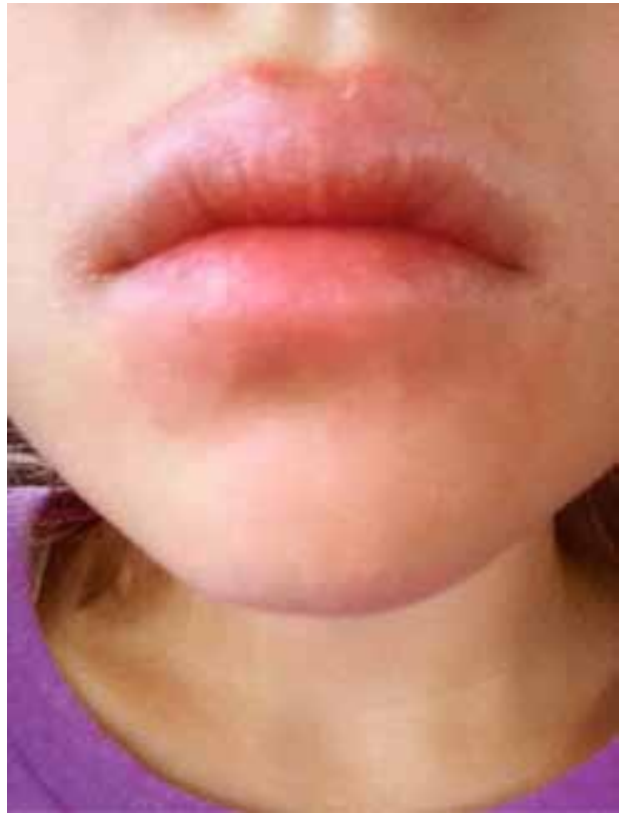

$\sigma$

Рис. 3. Девочка 10 лет, больная АтД: а - до лечения; б - на 5-й день терапии 0,03\% такролимусом 
индекса SCORAD с $52(43,5 ; 75,5)$ до $11,7(0,0 ; 26,2)$ балла $(p<0.0001)$. Величина курсового расхода мази варьировалась от 45 до 340 г (медиана 120 г). Среднесуточный расход составил 4,9 $\pm 3,3$ г. Несмотря на то, что нежелательные явления в виде незначительного зуда и жжения при нанесении мази отмечались у 11 (73\%) больных, отмены терапии не потребовалось ни в одном случае. Указанные явления разрешались самостоятельно в течение нескольких дней.

Об эфрфективности нового средства наружного действия для лечения АтД мази такролимус свидетельствует не одно сообщение и в отечественной $[13,14]$, и в зарубежной литературе $[10,15]$. По нашим данным, положительные результаты, характеризующиеся уменьшением выраженности клинических проявлений в виде эритемы, папулезной инфильтрации, сухости и шелушения, а также субъективных жалоб на зуд и нарушение сна, констатированы у всех больных в обеих группах. При этом клиническое выздоровление достиг- нуто у $30 \%$ детей и $40 \%$ взрослых пациентов. Нами отмечено быстрое наступление положительной динамики уже после первой недели лечения в группе пациентов детского возраста, что согласуется с результатами S. Kang и соавт. [16]. В группе взрослых больных уменьшение величины медианы индекса SCORAD более чем в 2 раза констатировано на 14-й день лечения. После лечения сравнение значений величин индекса SCORAD в обеих группах статистически значимых различий не выявило. Медиана индекса SCORAD в группе детей составляла $14(3,5 ; 22,1)$ баллов, в группе взрослых $-11,7(0,0 ; 26,2)$ балла $(p \geq 0,05)$.

\section{Заключение}

Высокая эффективность мази Протопик и отсутствие побочных эфрфектов, характерных для топических кортикостероидных препаратов, значительно расширяют возможности лечения пациентов с тяжелыми формами атопического дерматита. I

\section{Литература}

1. Kubanova A.A. Analysis of the epidemiological situation with the morbidity of STD as well as skin and hypoderm diseases among the population of the Russian Federation according to the official state statistics. Vestn dermatol veneroli 2008; (5): 8-18. [Kyбанова А.А. Анализ эпидемиологической ситуации по заболеваемости инфекциями, передаваемыми половым путем, болезнями кожи и подкожной клетчатки населения Российской Федерации, по данным официальной государственной статистки. Вестн дерматол и венерол 2008; (5): 8-18.]

2. Williams H.C., Atopic Dermatitis. The epidemiology, Causes and Prevention of Atopic Eczema. Cambridge University Press, Cambridge, UK, 2000.

3. Hanifin JM, Cooper KD, Ho VC, et al. Guidelines of care for atopic dermatitis, developed in accordance with the American Academy of Dermatology (AAD) American Academy of Dermatology Association «Administrative Regulations for Evidence-Based Clinical Practice Guidelines». J Am Acad Dermatol. 2004; 50 : $391-404$.

4. Emelyanov A.V.,Monakhov K.H. Topical corticosteroids in therapy allergic dermatitis: value of extra genomic effect. Vestn dermatol i venerol. 2002; ( 3): 59-61. [Емельянов А.В., Монахов К.Н. Топические кортикостероиды в терапии аллергодерматозов: значение внегеномного эффекта. Вестн дерматол и венерол 2002; (3): 59—61.]
5. Kubanova A.A. Samsonov V.A. Averbakh EV et al. Elokom in treatment of patients with psoriasis and atopic dermatitis. 1996; (5): 10—12. [Кубанова А.А., Самсонов В.А., Авербах Е.В., Тимошин Г.Г. Элоком в лечении больных псориазом и атопическим дерматитом. Вестн дерматол и венерол 1996; (5): 10 - 12.]

6. Grebenyuk V.N., Balabolkin I.I. Progress in topical steroids therapy of atopic dermatitis at children.Pediatrics. 1998; (5): 88-91. [Гребенюк В.Н., Балаболкин И.И. Прогресс в наружной кортикостероидной терапии атопического дерматита у детей. Педиатрия. 1998; (5): 88-91.]

7. Berth-Jones J, Damstra RJ, Golsch S et al. Twice weekly fluticasone propionate added to emollient maintenance treatment to reduce risk of relapse in atopic dermatitis: randomised, double blind, parallel group study. BMJ 2003; 326: 1367

8. Leung DY, Hanifin JM et al. Disease management of atopic dermatitis: a practice parameter. Ann Allergy Asthma Immunol 1997; 79: 197—211.

9. Satoshi Takeuchi, M.D., Ph.D., Hidehisa Saeki, M.D. Ph.D.2, Shoji Tokunaga. A Randomized, Open-Label, Multicenter Trial of Topical Tacrolimus for the Treatment of Pruritis in Patients with Atopic Dermatitis.Ann Dermatol 2012: 24: (2): 144-150.

10. Reitamo S., Rustin M., Ruzicka T., Cambazard F., Kalimo K., Friedmann P.S. et al; European Tacrolimus Ointment Study Group. Efficacy and safety of tacrolimus ointment compared with that of hydrocortisone butyrate ointment in adult patients with atopic dermatitis. J Allergy Clin Immunol 2002; 109: 547-555.
11. Antonello Baldo, Mariana Cafiero, Paola Di Caterino. Tacrolimus ointment in the management of atopic dermatitis. Clin Cosmet Investi Dermatol 2009: 2: 1-7.

12. Japanese FK-506 Ointment Study Group. Phase III study of FK-506 (Tacrolimus) ointment in patients with atopic dermatitis -Comparison study with $0.12 \%$ betamethasone valerate ointment for trunk and extremities lesions-. Nishinihon J Dermatol 1997; 59: 870—879.

13. Korotky N.G., Tikhomirov A.A., Gamajunov B.N. et al. New potentialities of topical therapy of severe atopic eczema in children. Pediatricheskaja farmakologija 2011; (8): 6: 96-102. [Короткий Н.Г., Тихомиров А.А., Гамаюнов Б.Н. и др. Новые возможности наружной терапии тяжело протекающих форм атопического дерматита у детей. Педиат фармакол 2011; 8: 6: 96-102.]

14. Kungurov N.V., Kohan M.M., Keniksfirst Y V et al. Proactive external therapy of children and adults, suffering from atopic dermatitis - new effective tactic approach Vestnik dermatologii i venerologii. 2012; (3): 15-120. [Кунгуров Н.В., Кохан М.М., Кенинксфест и др. Проактивная наружная терапия больных атопическим дерматитом детей и взрослых — новый, эффективный тактический подход. Вестн дерматол и венерол 2012; 3: 15-120.

15. R.S. Kirsner, M.P. Heffernan. Safety and Efficacy of Tacrolimus Ointment Versus Pimecrolimus Cream in the Treatment of Patients with Atopic Dermatitis Previously Treated with Corticosteroids. Acta Derm Venereol 2010; 90: 58-64.

16. Kang S, Paller A, Soter N. Safe treatment of head/neck $A D$ with Tacrolimus ointment. J of Dermatological Treatment 2003; 14, 86-94.

об авторах:

Д.В. Прошутинская - д.м.н, ведущий научный сотрудник, заведующая отделением детской дерматологии ФГБУ «ГНЦДК»

Минздрава России, Москва

М.М. Бутарева — к.м.н., заведующий дневным стационаром ФГБУ «ГНЦДК» Минздрава России, Москва

Л.А. Иноятова — врач-дерматовенеролог отделения детской дерматологии, аспирант ФГБУ «ГНЦДК» Минздрава России, Москва 